Trauma Berufskrankh 2017· [Suppl 1]: 19:S98-S100 DOI 10.1007/s10039-016-0182-1

Online publiziert: 25 . Juli 2016

(c) Springer-Verlag Berlin Heidelberg 2016

CrossMark

\section{Alexander Woltmann}

Allgemein- und Traumachirurgie, BG-Unfallklinik Murnau, Murnau, Deutschland

\section{Definition}

Das abdominelle Kompartmentsyndrom ist definiert als eine progrediente Erhöhung des intraabdominellen Druckes mit Dysfunktion der lebenswichtigen Organe und venöser Perfusionsstörung $[1,8,9]$. Es gehört in die Gruppe der Kompartmentsyndrome des Körpers, tritt vorwiegend nach Trauma auf und wurde das erste Mal durch Meldrum und Moore 1997 [6] definiert. Zum Vollbild gehören die Erhöhung des Blasendruckes auf über $20 \mathrm{cmH}_{2} \mathrm{O}$, Erhöhung des Beatmungsdruckes auf über $40 \mathrm{mmHg}$, Erniedrigung des Horowitz-Quotienten unter 150 Torr, Katecholamin-bedürftige Kreislaufinstabilität und Urinausscheidung unter $0,5 \mathrm{ml} / \mathrm{kg}$ pro Stunde. Das abdominelle Kompartmentsyndrom ist damit ein vital bedrohliches chirurgisches Krankheitsbild.

\section{Ätiologie}

Man unterscheidet die primären von den sekundären abdominellen Kompartmentsyndromen. $\mathrm{Zu}$ den primären Formen zählen die Verletzungen mit intra- oder retroperitonealen Blutungen sowie Darmschwellungen als Ganzkörper-Ischämie-Reperfusionssyndrom nach hämorrhagischem Schock, nach Schädel-Hirn-Trauma oder Wirbelsäulenverletzungen.

$\mathrm{Zu}$ den sekundären abdominellen Kompartmentsyndromen zählen die intensivmedizinisch induzierten intraabdominellen Druckerhöhungen im Rahmen einer Katecholamin- und Volumentherapie, die aufgrund von schwerwiegenden Verletzungen erforderlich sind.
Die Inzidenz wird nach Malbrain et al. $[4,5]$ mit 5-11\%, nach Meldrum et al. [6] mit $14 \%$ nach abdominellem Trauma angegeben. Die Prävalenz beträgt nach Ertel und Trentz [2] 5,5\% nach abdominellem und Beckentrauma, die Mortalität 35 \%.

\section{Symptomatik}

Das abdominelle Kompartmentsyndrom äußert sich durch eine Umfangvermehrung des Bauches sowie eine erhöhte Bauchdeckenspannung. Hinzu kommen die Darmparalyse [7], die Oligurie und das Steigen des Beatmungsdruckes. Im Bereich der Lunge zeigen sich ein Zwerchfellhochstand, basale Atelektasen, eine verminderte Compliance, eine Hypoxie und Hyperkapnie. Am Herzen äußert sich das abdominelle Kompartmentsyndrom mit einer Rechtsherzbelastung, einem verminderten venösen Rückfluss (Preload), einem verminderten Herzzeitvolumen und einer Linksherzinsuffizienz. An der Niere kommt es zur Verminderung des venösen Abstroms mit sekundär abnehmender arterieller Perfusion und damit induzierter Oligo- und Anurie. Am Gehirn ist ebenfalls der venöse Abstrom behindert mit Anstieg des intrakraniellen Druckes und verminderter zerebraler Perfusion [3]. Im Gastrointestinaltrakt kommt es
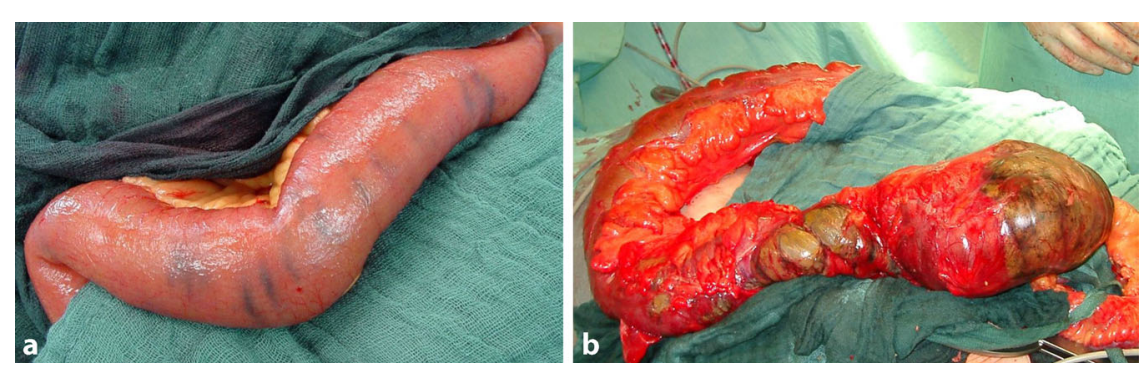

Abb. $1 \Delta$ a Beginnende und b vollständige Darmwandnekrosen am a Dünn- und b Dickdarm zur Verminderung der Durchblutung im Splanchnikusgebiet mit anaerobem Stoffwechsel und Azidose. Die Darmbandpermeabilität steigt, und es kommt zur bakteriellen Translokation und Endotoxinämie (• Abb. 1).

\section{Diagnostik}

Zur Diagnostik gehören die Sonographie mit Nachweis von Pendelperistaltik, die Röntgenübersichtsaufnahme des Abdomens und eine Linksseitenlage, in der Spiegel nachgewiesen werden können, sowie die CT-Diagnostik, in der die Spiegel ausgemessen werden und ggf. Lufteinschlüsse in der Darmwand oder der Leber zu sehen sind. Der intraabdominelle Druck kann als Goldstandard über den Harnblasenkatheter gemessen werden. Normalerweise besteht ein atmosphärischer Druck, postoperativ kann ein Druck von 5-12 $\mathrm{cmH}_{2} \mathrm{O}$ gemessen werden. Beim beatmeten Patienten misst man endexspiratorisch. Werte über $20 \mathrm{cmH}_{2} \mathrm{O}$ sind pathologisch.

\section{Therapie}

Die Indikation zur Laparotomie orientiert sich an den gemessenen intraabdominellen Druckwerten (• Tab. 1) und wird anhand der Dynamik des Krank- 


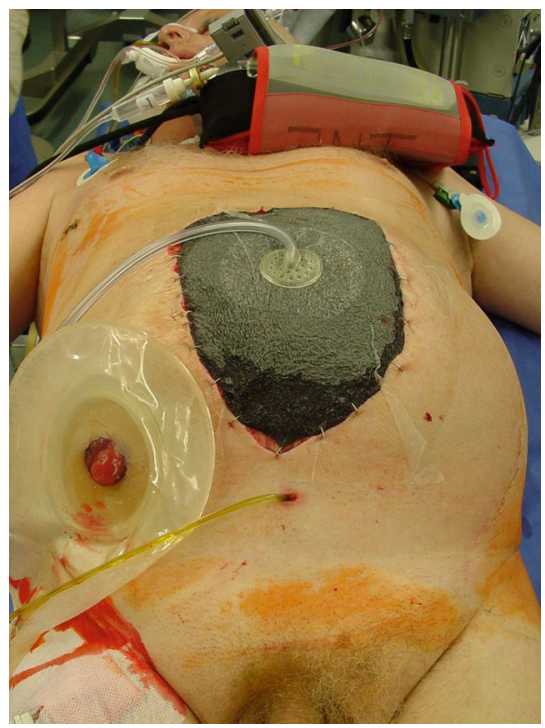

Abb. 2 ॥ Entlastungslaparotomie, lleostoma und Vakuumversiegelung durch Schwamm und Folie

heitsbildes gestellt. Hierzu ist es wichtig, die Bauchdeckenspannung, die Darmgeräusche, den klinischen Zustand mit Fieber, ggf. Reflux und Darmparalyse mit Ileussymptomatik zu beurteilen. Hinzu kommen die Laborwerte des Laktates, der Leukozyten, des C-reaktiven Proteins (CRP), des Procalcitonins (PCT) und der Thrombozyten. Auch bei vermehrtem Hirndruckzeichen, sich verschlechternder Beatmungssituation und ansteigendem Katecholaminbedarf sowie einsetzender Anurie ist die Indikation zur Laparotomie zu stellen.

Bei der Laparotomie ist der Darm auf Nekrosen zu untersuchen. Liegen keine Nekrosen vor, kann bei Dünndarmmileus eine retrograde Dekompression die Situation deutlich verbessern. Liegt bereits eine Dünndarmsegmentnekrose vor, ist dieses $\mathrm{zu}$ resezieren. Hier kann dann eine End-zu-End-Anastomose oder auch der Blindverschluss erfolgen. Liegt am Kolon eine erhebliche Distension vor und zeigt die Wand noch keine Nekrosen, kann das Absaugen über eine Appendektomie effektiv zur Dekompression beitragen. Sind bereits Nekrosen vorhanden, erfolgt die Kolonsegmentresektion, in der Regel die Rechtskolonresektion, da das Rechtskolon am häufigsten betroffen ist. Nützlich ist die Anlage eines Ileostomas, die den pseudoobstruktiven Stopp aufhebt

Trauma Berufskrankh 2017 · [Suppl 1]: 19:S98-S100 DOI 10.1007/s10039-016-0182-1

(c) Springer-Verlag Berlin Heidelberg 2016

\section{A. Woltmann}

\section{Abdominelles Kompartmentsyndrom}

\section{Zusammenfassung}

Das abdominelle Kompartmentsyndrom tritt vorwiegend nach Trauma auf. Ursachen können sein: Die intra- oder retroperitoneale Blutung, eine diffuse Vermehrung der subperitonealen interstitiellen Flüssigkeit („,capillary leak syndrome") oder die intestinale Pseudoobstruktion mit Paralyse und Distension, insbesondere des Rechtskolons Typ Ogilivie (1948) u. a. Alle vorgenannten Situationen können zu einer intraabdominellen, vital bedrohlichen Druckerhöhung führen, die mittels Druckabnehmer im Blasenkatheter gemessen werden und zur Entlastung durch Laparotomie zwingen kann. Die Maßnahme kann den Teufelskreis eines Multiorganversagens durchbrechen.
Nach einigen Tagen der Behandlung mit offenem Abdomen sollen der Cavarückfluss, der kardiale Auswurf, der Gasaustauch und die Nierenausscheidung verbessert werden. Nützlich ist die Anlage eines protektiven lleostomas, über das die Darmpassage und die intestinale Durchblutung kontrolliert werden können. Am Ende der Behandlung stehen die Rekonstruktion der Passage und der Bauchdecke, die ggf. auch erst Monate später sekundär herbeigeführt werden kann.

\section{Schlüsselwörter}

Intraabdominelle Druckerhöhung · Laparotomie $\cdot$ lleostoma $\cdot$ Multiorganversagen . Trauma

\section{Abdominal compartment syndrome}

\section{Abstract}

Abdominal compartment syndrome is predominantly caused by trauma. Intraperitoneal or retroperitoneal bleeding, visceral capillary leak syndrome or intestinal pseudoobstruction due to sympathetic deprivation with paralysis and distension, particularly Ogilvie syndrome of the right colon are possible underlying mechanisms. All of these situations can lead to intra-abdominal, lifethreatening hypertension, which can be measured by a pressure detector in a urinary bladder catheter, in concert with multiple organ failure. Decompressive laparotomy is a useful procedure to break this vicious circle.
After some days of treatment with an open abdomen, the caval preload, cardial output, pulmonary function and renal excretion should recover. The placement of a protective ileostoma can be useful to control intestinal passage and vascular perfusion. The final procedure in the treatment is reconstruction of the intestinal passage and the abdominal wall, which can be performed some months later if necessary.

\section{Keywords}

Intra-abdominal hypertension - Laparotomy . lleostomy $\cdot$ Multiple organ failure $\cdot$ Trauma und eine Kontrolle der Passage und der Durchblutung bettseitig erlaubt. Der Bauchdeckenverschluss erfolgt temporär mit einem folienarmierten Bauchtuch und einer Vakuumversiegelung über einem Schwamm (• Abb. 2). Der Sog wird niedrig reguliert auf $25-50 \mathrm{mmHg}$ mit einer intermittierenden Sogwirkung, z. B. 1 min saugen und $5 \mathrm{~min}$ Pause. Relaparotomien zur Revision bieten sich nach 2 bis 5 Tagen an. Sobald die Bauchdeckenspannung dies erlaubt, kann der sekundäre Bauchdeckenverschluss herbeigeführt werden. Die Ileostomarückverlagerung ist frühestens nach 9 Wochen $\mathrm{zu}$ empfehlen. Sollte ein Laparostoma nicht sekundär direkt verschlossen werden können, eignen sich die Bauchdeckenverstärkung durch Vicrylnetz und das Decken des Defektes mit Meshgraft.

\section{Eigene Ergebnisse}

In einer Beobachtungszeit über 3 Jahre aus den Jahren 2009 bis 2011 konnten an der Unfallklinik Murnau bei 42 Patienten mit Poly- und Abdominaltrauma mit einem Abbreviated Injury Scale über 37 Verletzte mit abdominellem Kompartmentsyndrom differenziert werden, was einer Prävalenz von $17 \%$ entspricht. Die Ursache waren Verkehrsunfälle, alle Patienten mussten entlastungslaparotomiert 
Tab. 1 Schweregrade des abdominellen Kompartmentsyndroms nach Meldrum und Therapie

\begin{tabular}{|lll}
\hline Grad & Blasendruck $\left[\mathbf{C m H}_{\mathbf{2}} \mathbf{O}\right]$ & Therapie \\
\hline I & $10-15$ & Normovolämie aufrechterhalten \\
\hline II & $16-25$ & Offensive Volumentherapie \\
\hline III & $26-35$ & Dekompression \\
\hline IV & $>35$ & Dekompression und Reexploration \\
\hline
\end{tabular}

werden. Der Injury Severity Score betrug im Mittelwert 38, das Alter 53 Jahre, die Intensivbehandlungszeit $28 \mathrm{Ta}$ ge, die Hospitalisationszeit 50 Tage. Im Mittel waren 5 abdominelle Revisionen erforderlich. An Begleitverletzungen lagen 6-mal Thorax- und Extremitätenverletzungen, 5-mal Beckenverletzungen, in 2 Fällen ein Schädel-Hirn-Trauma und bei 1 Patienten ein Wirbelsäulentrauma vor. Bei 2 von 7 Patienten musste ein Ileostoma angelegt werden. Die Mortalität dieser Patientengruppe betrug $42 \%$ (3 von 7 Patienten). Die spätere Rekonstruktion der Bauchdecke erfolgte ebenfalls bei 3 von 7 Patienten durch eine Netzaugmentation.

\section{Fazit für die Praxis}

- Beim abdominellen Kompartmentsyndrom handelt es sich um eine fatale/vitale Bedrohung.

- Die chirurgische Dekompression (Entlastungslaparotomie) und Ableitung über ein Ileostoma sind das Mittel der Wahl.

- Später kann die Rekonstruktion der Darmpassage und der Bauchwand vorgenommen werden.

\section{Korrespondenzadresse}

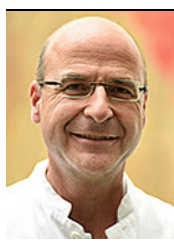

Prof. Dr. A. Woltmann

Allgemein- und Traumachirurgie, BG-Unfallklinik Murnau

Prof. Küntscher-Str. 8, 82418 Murnau, Deutschland woltmann@bgu-murnau.de

\section{Einhaltung ethischer Richtlinien}

Interessenkonflikt. A. Woltmann gibt an, dass kein Interessenkonflikt besteht.
The supplement containing this article is not sponsored by industry.

\section{Literatur}

1. Cheatham ML (2009) Abdominal compartment syndrome:Pathophysiologyand definitions. Scand JTrauma ResuscEmerg Med 17(1):10. doi:10.1186/ 1757-7241-17-10

2. ErtelW, Trentz O (2001) Das abdominelle Kompartmentsyndrom. Unfallchirurg 104:560-568

3. Joseph DK, Dutton RP, Aarabi B, Scalea TM (2004) Decompressive laparotomy to treat intractable intracranial hypertension after traumatic brain injury. JTrauma 57:687-695

4. Malbrain MLNG, Chiumello D, Pelosi P, Bihari D, Innes R et al (2005) Incidence and prognosis of intraabdominal hypertension in a mixed population of critically ill patients: A multiplecenter epidemiological study. Crit Care Med 33:315-322

5. Malbrain MLNG, Deren D, Potter TJR de (2005) Intraabdominal hypertension in critically ill: It is time to pay attention. Curr Opin Crit Care 11:156-171

6. Meldrum DR, Moore FA, Moore EE, Franciose RJ, Sauaia A, Burch JM (1997) Prospective characterization and selective management of the abdominal compartment syndrome. Am J Surg 174:667-672

7. Ogilvie H (1948) Large-intestine colic due to sympatheticdeprivation-anewclinical syndrome. BrMed J 9:671-673

8. Parsak CK, Seydaoglu G, Sakmann G, Acarturk TO, Karakoc E, Hanta I, Alparslan AH, Satar $S$ (2008) Abdominal compartment syndrome: current problems and new strategies. World J Surg 32:13-19

9. Sugrue M (2005) Abdominal compartment syndrome. Curr Opin Crit Care 11:333-338 